\title{
Morphological evolution of a single crystal silicon battery electrode during lithiation and delithiation: An operando phase-contrast imaging study
}

\author{
Arne Ronneburg ${ }^{1,2}$, Markus Osenberg ${ }^{3}$, Kang Dong ${ }^{3}$, André Hilger ${ }^{3}$, Eneli Härk ${ }^{1}$, Luca Silvi ${ }^{1}$, Ingo Manke ${ }^{3}$, Matthias \\ Ballauff ${ }^{1,2}$ and Sebastian Risse ${ }^{1^{*}}$ \\ ${ }^{1}$ Helmholtz-Zentrum Berlin, Institute for Electrochemical Energy Storage, Hahn-Meitner Platz 1, 14109 Berlin, Germany \\ ${ }^{2}$ Humboldt-University Berlin, Institute of Physics, Zum Großen Windkanal 6, 12489 Berlin, Germany \\ ${ }^{3}$ Helmholtz-Zentrum Berlin, Institute of Applied Materials, Hahn-Meitner Platz 1, 14109 Berlin, Germany
}

KEYWORDS: Silicon anode, Lithium-ion battery, operando analysis, X-ray imaging, structure formation, energy storage, impedance spectroscopy

ABSTRACT Operando phase-contrast radiography combined with impedance spectroscopy and electron
microscopy is applied to study the morphological changes in a lithium-silicon cell over several cycles.
The single-crystal silicon (100) surface is employed as a working electrode. A checkerboard-like cracking
pattern aligned with the crystallographic axis is formed in the 4th cycle during the second step of the
delithiation. With the crack position kept fixed, this crack pattern vanishes during lithiation and gets more
pronounced in the subsequent cycles. This pattern forms just after the first delithiation peak that
corresponds to the decomposition of the highly lithiated phase. It vanishes during lithiation in the
potentiostatic step. Therefore, capacity-limited cycling, which partly maintains the highly lithiated phase,
may avoid the formation of fractures. The surface area of the crack cells remains almost constant with
increasing cycle numbers, and no electrochemically inactive sites were observed. INTRODUCTION

Lithium-ion batteries are commonly used in daily life and represent the state-of-the-art battery system $^{1,2}$. For this battery type, graphite is the mainly used anode with a theoretical capacity of $372 \mathrm{mAh}$ $\mathrm{g}-1$, which limits the overall capacity ${ }^{3}$. In contrast, silicon has a theoretical specific capacity of $4200 \mathrm{mAh}$ g-1 and, therefore, can replace the graphite anode to increase the overall capacity ${ }^{4}$. This high capacity, combined with the nontoxicity and abundance of silicon, has led to intense research in this field ${ }^{2-4}$. However, the major drawback of this anode material is the volume change of more than $360 \%$ during cycling. ${ }^{5}$. This expansion creates internal stress in the silicon and introduces cracks within the electrode, which leads to a noticeable capacity fading ${ }^{6}$.

Many studies were conducted to gain deeper insights into the fading mechanisms of silicon electrodes. Diffraction experiments showed the conversion of crystalline silicon into an amorphous phase with different lithium concentrations, but the highly lithiated phase, $\mathrm{Li}_{15} \mathrm{Si}_{4}$, recrystallizes again ${ }^{7}$. Electron microscopy was used to visualize the lithiation process on the nanoscale and revealed a two-step lithiation mechanism $^{8}$. Different morphologies were studied with electron microscopy, such as nanowires ${ }^{9}$, nanoparticles $^{10,11}$, or porous nanostructures ${ }^{12,13}$. The lithiation is accompanied by a volume expansion, which was shown by theoretical calculations ${ }^{14-16}$ and quantified with neutron reflectometry by Jerliu et al. ${ }^{17}$. The volume expansion is accompanied by tensile or compressive stress, depending on the lithiation degree. For nano-sized particles, this was shown through diffraction measurements and Raman spectroscopy 18,19. Theoretical calculations linked the lithiation-induced stress with the fracture of different geometries and proposed an upper limit of $150 \mathrm{~nm}$ for spheres to avoid fracturization ${ }^{20}$. Indeed, this theoretical limit was confirmed by McDowell et al. and Liu et al., who observed the fracturization process for nano-sized particles using electron microscopy ${ }^{10,11}$. Similar observations were made on the macroscale using radiography or tomography, visualizing the volume expansion and fracturization of micron-sized particles ${ }^{21-27}$. The volume expansion during lithiation damages the host matrix, which leads to a loss of electrical connection and delamination ${ }^{22,25,27}$. Inhomogeneous lithiation was observed using complementary X-ray and neutron imaging ${ }^{26}$ as well as the creation of porosity within the electrode ${ }^{27}$. The studies mentioned above 
demonstrate a strong dependence of the lithiation process on the electrode morphology ${ }^{2,22-24}$. The interplay between binder, conductive agent, and size distribution of the particles increases the complexity of the system and complicates a mechanistic understanding of the lithiation process. Up to now, no consensus about the aging mechanisms has been achieved yet.

In contrast to the enormous work on particle-based anodes, much less research was done using single crystalline silicon electrodes, although it is a well-defined model electrode ${ }^{28-34}$. Single-crystal silicon allows us to study the lithiation process in a system without any binder or conductive agent. Operando neutron and X-ray reflectometry reveal the depth-dependent lithium concentration over several cycles, but no fractures were observed due to experimental limitations ${ }^{32-34}$. The mechanical stress within the (100) silicon was determined by Chon et al. depending on the cell voltage ${ }^{29}$, whereas Jana et al. elucidated the cycle-dependent change of the residual stress within the (100) Si-crystal ${ }^{28}$. An ex-situ electron microscopy study revealed cracking of (100) silicon after several cycles ${ }^{30}$, whereas the crack propagation depends on the crystal orientation ${ }^{31,35}$. However, the dependency of the fractures on the lithiation degree and their evolution is hardly understood. More operando studies aiming at the macroscale could improve the mechanistic understanding of the cracking process.

A suitable technique to analyze cracks and material boundaries within a specimen is phase-contrast imaging ${ }^{36-38}$. This technique additionally employs the phase shift of the incident $\mathrm{x}$-ray beam to visualize a specimen, whereas the traditional attenuation-based imaging uses the intensity decrease solely. Therefore, refraction occurs at the boundaries of different refractive indices. This effect leads to a slight deviation of the X-ray paths (propagation-based phase-contrast X-ray imaging) ${ }^{36,38}$. The resulting dippeak pair in the X-ray intensity causes a sharp contrast of interfaces within the sample ${ }^{37}$. For this reason, a monochromatic, parallel, and coherent beam is required. For elements with a low atomic number, the ratio of phase shift coefficient to the absorption coefficient is approximately three orders of magnitude. The phase shift and absorption coefficient correspond to the real and imaginary part of the refractive index, respectively. Therefore, imaging under the X-ray phase-contrast mode is highly suitable to analyze specimens with light elements such as silicon or lithium ${ }^{36}$.

Here we present, to the best of our knowledge, the first operando X-ray phase-contrast imaging study of the electrochemical lithiation process of (100) crystalline silicon (Fig. 1). A voltage-controlled cell operation, consisting of a linear sweep, followed by a potentiostatic step, was chosen to promote the formation of different lithiated silicon species and their influence on the electrode morphology. For instance, the formation of the highly-lithiated phase occurs only at voltages $<70 \mathrm{mV}$. ${ }^{32}$ Phase-contrast imaging is susceptible to material boundaries and, therefore, well suited to study the fracturization process ${ }^{36}$. In total, eight cycles were studied. Roughly 150 images were recorded per cycle, which allows us to study the fracturization process under operando-conditions. Besides, focussed ion beam microscopy (FIB/SEM) is used to assign the observed characteristics to the specific electrode, since radiography creates a superimposed view of both electrodes. Additional in-situ impedance spectroscopy gives

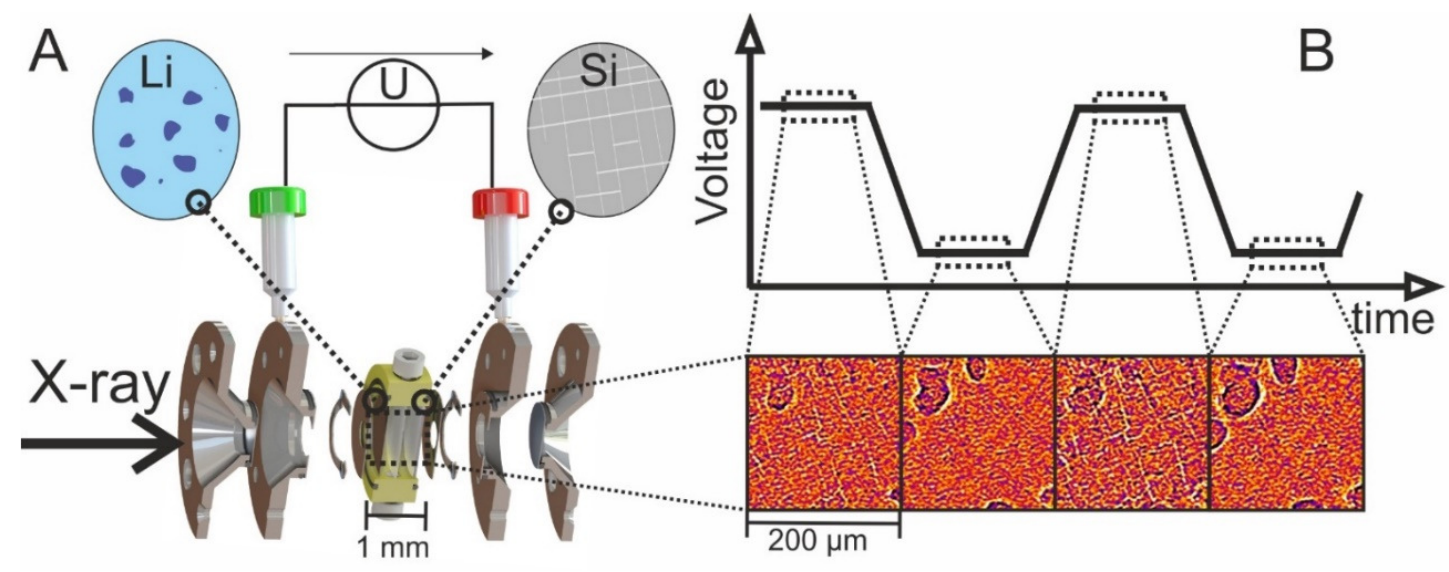

Fig 1: A) Schematic sketch of the operando setup. A lithium metal chip and a silicon wafer are used as electrodes. A potentiostat controls the cell voltage. The silicon cracks during delithiation. B) Schematic sketch of the applied voltage profile. At high voltages (delithiation), cracks become visible in the obtained radiograms. After lowering the voltage, 2 lithiation starts, and the cracks disappear. The sequence was repeated over eight cycles. X-rays generated from a synchrotron source (BAMline station at BESSY II) are used to elucidate the internal processes. The resulting image is based on the refraction of X-rays on material interfaces (phase-contrast imaging) 
information about the electrochemical properties of the system, and ex-situ Laue diffraction helps to correlate the observed morphology changes to the crystallographic axis. Therefore, we provide mechanistic insights into the voltage-dependent fracturization of silicon electrodes. First, the essential electrochemical performance of the experiment will be compared to systems described elsewhere ${ }^{4,30,39}$. Afterward, the results obtained by operando radiography will be discussed, followed by the observations from ex-situ studies, namely FIB/SEM and Laue diffraction.

\section{RESULTS AND DISCUSSION}

Analysis of the electrochemical performance

Figure 2 summarizes the acquired electrochemical results. The voltage excitation is indicated as the black dotted line shown in Fig 2A, whereas the solid red line shows the current response of the system. The cycle-dependent increase in current is caused by the successive increase in electrochemically active silicon. ${ }^{30}$. This is a consequence of the amorphization of silicon of prior cycles. As shown in figure 2B, the lithiation of the amorphous phase occurs already at $300 \mathrm{mV}^{7,34,40}$, while the lithiation of the crystalline phase in the first cycles starts at voltages lower than $100 \mathrm{mV}{ }^{4,7,30}$. Only the first cycle shows a peak at $1.4 \mathrm{~V}$ during voltage ramp down (inset in figure 2B). Therefore, this can be associated with the lithiation of the silicon oxide layer superimposed with the removal of adsorbed species. At $0.4 \mathrm{~V}$ and $0.6 \mathrm{~V}$, two delithiation peaks can be found, which shifts slightly to higher voltages (560 mV in the first cycle, 650 $\mathrm{mV}$ in the $8^{\text {th }}$ cycle for the second delithiation peak). Both peaks correspond to the delithiation of different lithium-silicon phases ${ }^{4,30,41}$. Up to the third cycle, both peaks are comparable in height; afterwards, the high voltage peak becomes significantly larger. Shi et al. ${ }^{30}$ also observed this effect in a similar experiment with a lower voltage rate of $0.1 \mathrm{mV} / \mathrm{s}$. This result indicates a diffusion limitation of the dissolution of the lowly lithiated silicon phase, as suggested by Pharr et $a l^{35}$. No off-set current, and no additional current peaks can be observed.

The charge balance in fig. $2 \mathrm{C}$ shows a constant irreversible charge loss of about $0.2 \mathrm{C}$ from the second cycle on. This discrepancy originates from parasitic side reactions associated with the solid-

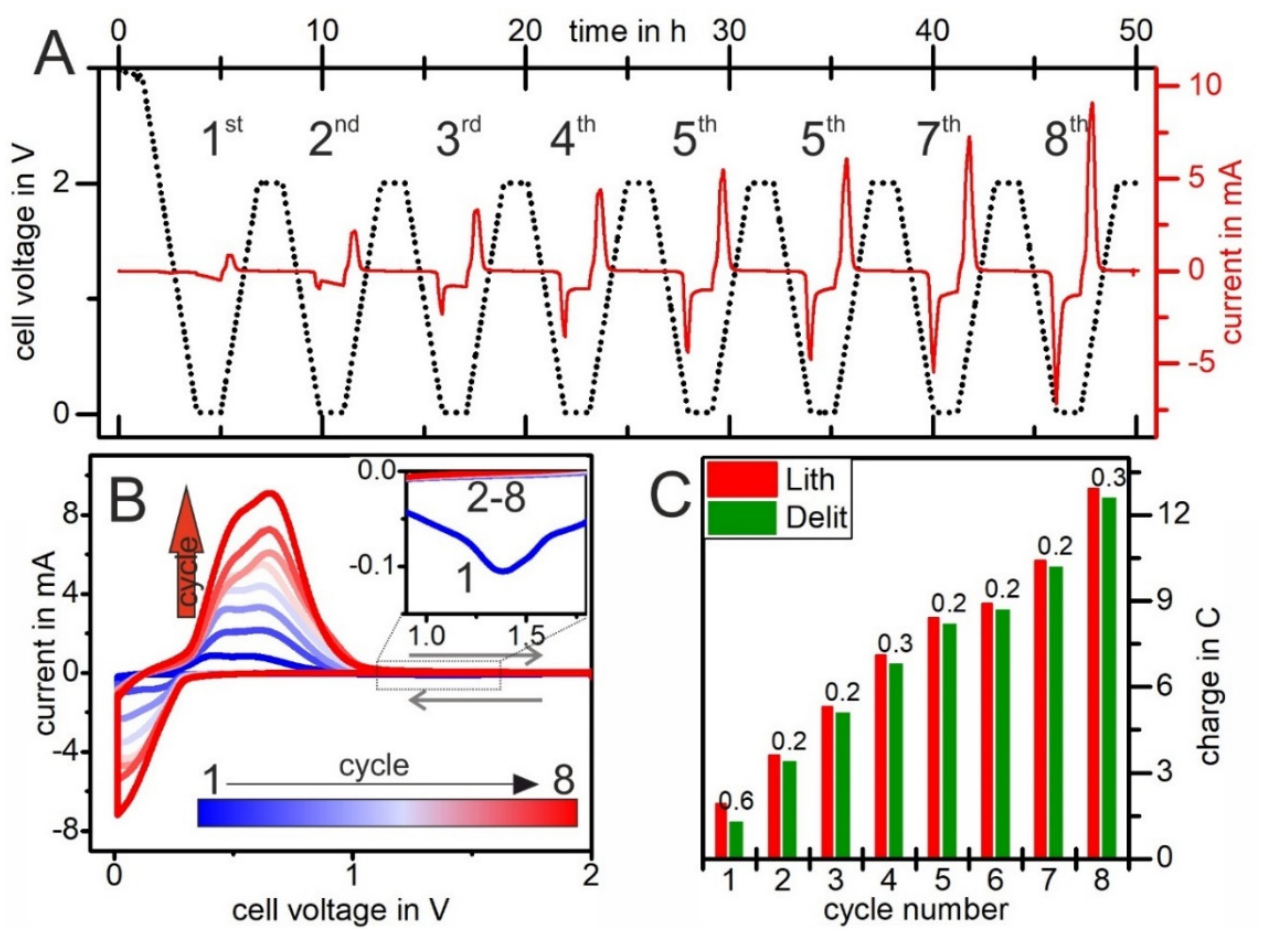

Fig. 2: A) Time-dependent cell voltage set by the potentiostat (dotted black line) and the corresponding current response (solid red line). B) Cyclic voltammetry plots for cycle 1 (red) to 8 (blue). The inset shows the region around the high voltage peak, which only occurs in the first cycle. The grey arrows indicate the direction of the voltage change. C) Comparison of lithiation (red) and delithiation (green) charge. The charge difference is nearly constant, except for the 3 first cycle. 
electrolyte-interphase layer (SEI). The first cycle shows a higher charge loss of $0.6 \mathrm{C}$ caused by a small peak around $1.4 \mathrm{~V}$ (inset Fig 2B). With cycling, the coulombic efficiency increases from 0.68 to 0.98 since the incorporated charge increases, but the irreversible charge loss stays constant. Most probably, the charge loss originates from parasitic side reactions such as the reformation of a SEI ${ }^{33,43,44}$. The acquired electrochemical data exhibit all significant features of the lithiation of silicon ${ }^{4}$. Therefore, the customdesigned operando-cell allows us to link the imaging results to the corresponding electrochemical processes.

Operando-results

Operando phase-contrast radiography was performed to link the electrochemical processes and structural changes within the cell. The X-ray beam penetrates several layers, including the metal lithium electrode, separator, and silicon electrode. Due to the high stability of the separator (Celgard 2700), no morphological changes of the separator within the eight cycles were observed. Therefore, the observed structural changes in the experiment are attributed only to both electrodes. The assignment of the observed morphology changes to the respective electrode was done by investigating the cycled silicon electrode with FIB/SEM. The circular structures are shown in Fig. 3 are located on the lithium electrode and are created through the lithium pitting process ${ }^{45}$. The straight lines can be found on the silicon electrode and originate from the crack pattern of the silicon caused by the volume expansion and the brittle nature of the electrode. The radiography images were processed to get a qualitative view of the morphology changes (see also experimental section). The full video file is available online. First, a single cycle will be discussed more in detail to correlate the occurrence of cracks within the silicon with the cell voltage. The $8^{\text {th }}$ cycle is chosen as an example since the cracks are most pronounced here (figure 3 ). Afterwards, evolution over many cycles will be discussed (figure 4).

The crack intensity of cycle eight is shown in the top row, the current in the middle, and the voltage is plotted in the bottom row of figure 3. Four radiograms are shown in the right part to demonstrate the status of the electrodes at specific voltages. The first image (A) shows the electrode during the lithiation step before the cracks start to vanish (around $120 \mathrm{mV}$ ). Once the voltage is lowered to $15 \mathrm{mV}$, the silicon electrode gets smooth, and the cracks are not visible anymore. Only the pits, formed during the lithium anode corrosion, remain in the field of view ${ }^{45}$. The voltage indicates the lithiation of crystalline silicon and the formation of the highly lithiated phase $\mathrm{Li}_{22} \mathrm{Si}_{5}{ }^{4}$. Therefore, the volume expansion of the silicon fills the interspaces. In literature, an anisotropic expansion of silicon is reported ${ }^{46,47}$, but not observed here since all cracks seem to vanish simultaneously. A possible reason is the limited in-plane-space within the silicon matrix, which hinders a deformation of the silicon patches. A total charge of $12.9 \mathrm{C}(7.9 \mathrm{C} \mathrm{cm}-2)$ was deposited within the silicon electrode, the majority during crack closure.

The inverse process is shown in the images $C$ and $D$, where the cracks reappear. Image $C$ shows the intermediate stage between the two delithiation current peaks. It is noteworthy to mention that

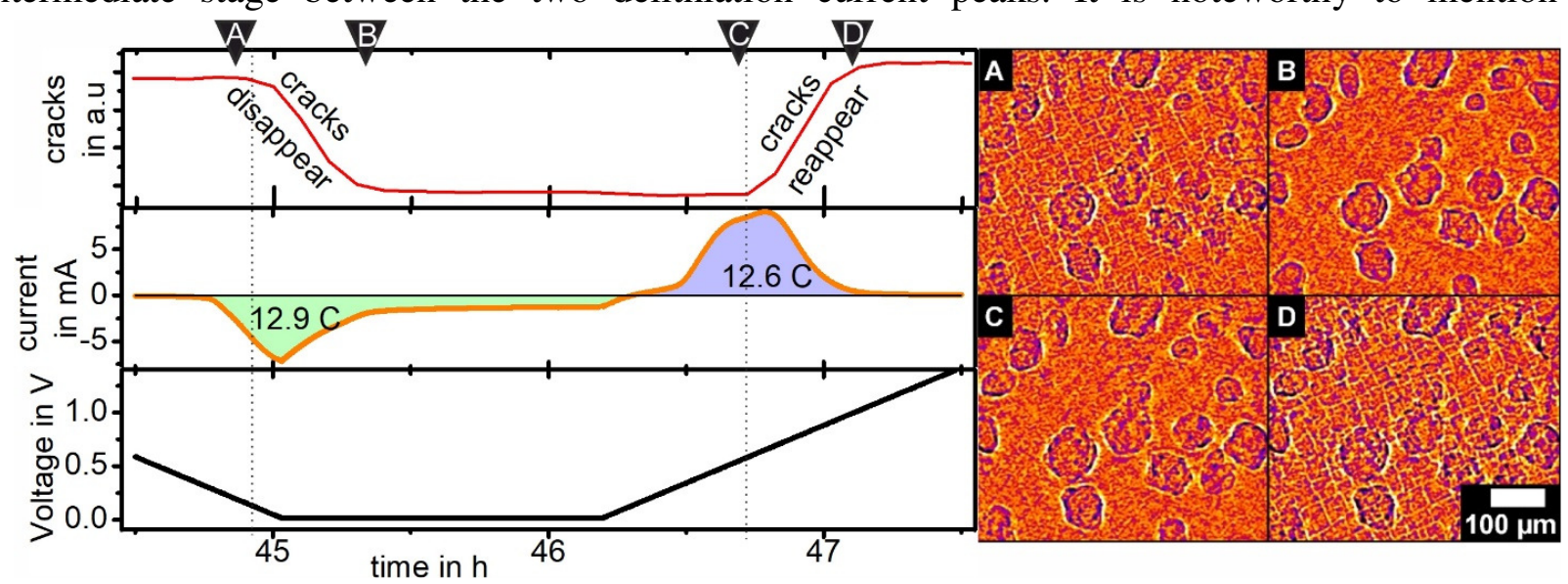

Fig. 3: Correlation of electrochemical data and structural change in the $8^{\text {th }}$ cycle. The crack intensity is shown on the top (left side). The current is plotted in the middle row, and the voltage profile is in the bottom row. Note that not the full cycle is shown but only the part where morphological changes occur. The respective radiograms assigned by the letters A-D are shown on the right. 
approximately half of the lithium was removed already from the silicon before the cracks became rapidly visible. This observation demonstrates that silicon can withstand a certain stress level before macroscopic effects become visible, which is the cracking of the brittle silicon. The current peak around $500 \mathrm{mV}$ is associated with the delithiation of the highly lithiated phase, whereas the peak at $650 \mathrm{mV}$ is ascribed to the complete lithium removal ${ }^{30,41,48}$. So, limiting the lithiation degree to a specific range might avoid the fracture of the silicon electrode surface, which was investigated already by Obrovac et al. in an ex-situ study ${ }^{40}$. In their work, they suggested a charge limited lithiation process to preserve high charge retention. The voltage-dependent morphology change of the first and seventh cycle, as well as the charged and discharged state of all cycles, is shown in the supporting information (Fig. S1)

Figure 4 summarizes the electrochemical data and the obtained crack intensities over the whole experiment. Cell voltage and current are shown in Fig. 4A and B, whereas 4C shows the crack intensity. Fig. 4D illustrates the results of the impedance spectroscopy at the end of each potentiostatic step. As already discussed, the current increases with the cycle number due to the ongoing amorphization of silicon 4,7. A slight increase in the crack intensity can be found in the first two cycles, but no fracturization pattern can be observed. Instead, the wetting process can be observed as well as a roughening of the surface. Both processes may lead to this artifact in the crack intensity plot within the first two cycles. The first cracks appear in the third delithiation and get more pronounced from cycle to cycle. This observation yields a possible explanation for the increase in the high voltage peak during the delithiation from the fourth cycle

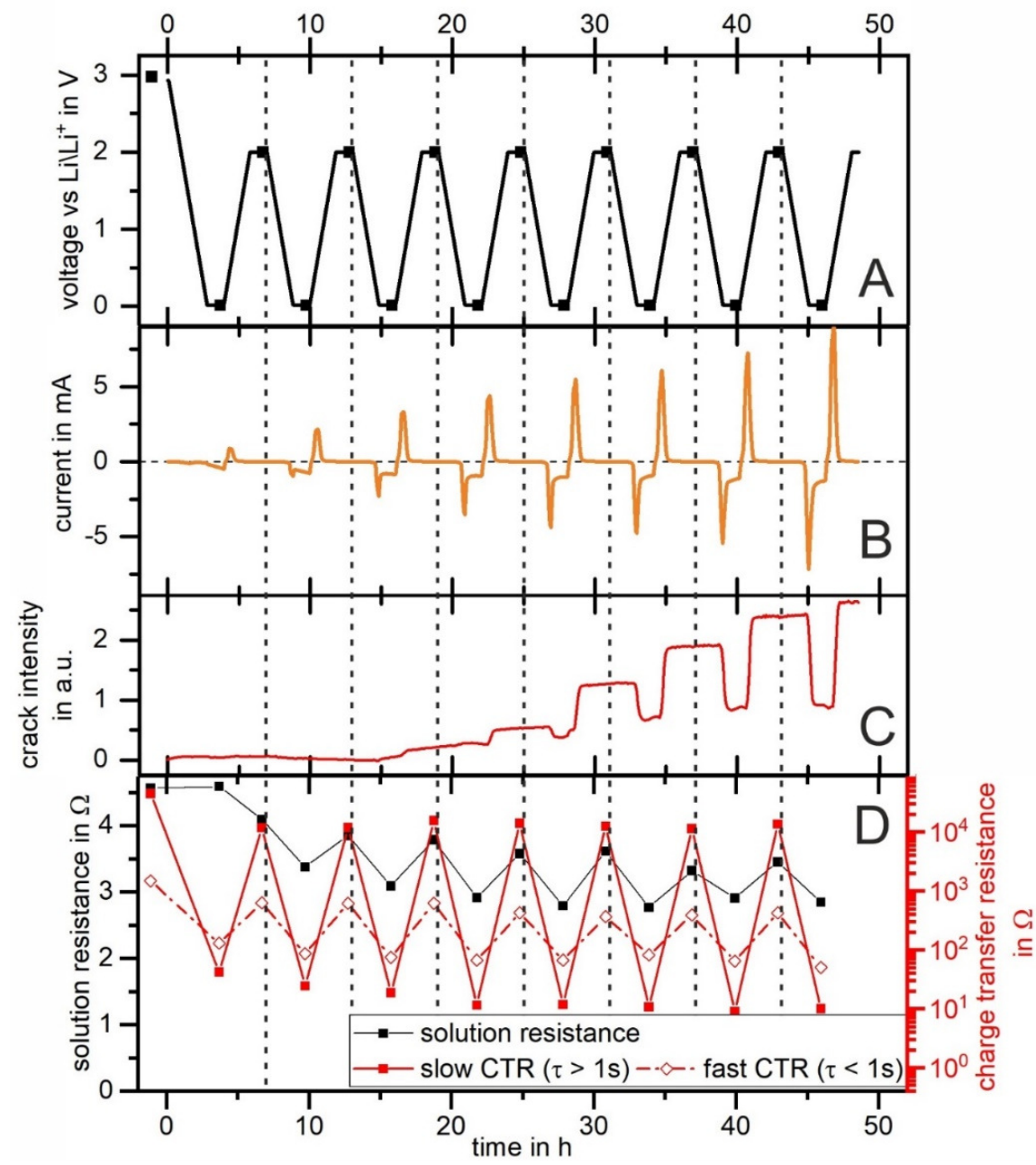

Fig. 4: A) Voltage profile of the cell during the experiment. The black squares denote the measurement of an EIS B) Corresponding current of the cell. C) Crack intensity obtained from processed radiograms. The crack intensity increases in the delithiated state and decreases in the lithiated state. The intensity originates from the phase-contrast evolving at phase boundaries. D) Solution resistance and charge transfer resistances for fast $(\tau<1 \mathrm{~s})$ and slow $(\tau>1 \mathrm{~s})$ charge transfer processes. Clearly, alternating behavior can be found. The used equivalent circuit is shown in fig. S2, together with the 5 obtained data. The time axis is shared by all four graphs. Each dashed line indicates the end of a cycle. 
on. The cracking of the electrode increases the electrochemically active area. Therefore, the diffusion limitation of the lithium ions in the amorphous silicon phase, as suggested by Pharr et al. ${ }^{35}$, is circumvented by now available shorter diffusion paths to the silicon/electrolyte interphase.

The crack intensity decreases during the lithiation process due to the volume expansion of the silicon electrode, especially during the potentiostatic step. Both electrochemically induced morphology changes take approximately 40 minutes. The crack intensity increases again during delithiation between the two cathodic peaks at approximately $600 \mathrm{mV}$. Here the volume shrinks, and fractures within the brittle silicon are revealed. The crack intensity increases with cycle number since the area of the silicon surface covered with fractures increases, and the contrast of the individual cracks increases with cycling. This represents the mechanical aging of the electrode. However, no inactive spots on the silicon electrode were observed during the operando imaging experiment, which would be indicated by certain places that do not undergo morphological changes anymore. The irreversible charge loss is shown in fig. $2 \mathrm{C}$ is therefore caused by parasitic side reactions associated with the $\mathrm{SEI}^{33,43,44}$.

A lab-based galvanostatic cycling experiment in a standard coin cell (CR2032) shows that even after 40 cycles, the charge retention of this system stays close to unity, and no electrode failure was observed (Fig. $\mathrm{S} 3$ in the supporting information). The spallation of active material from the electrode would decrease the coulombic efficiency since the fractures form after half of the charge is extracted. However, the spallation of silicon after complete delithiation cannot be excluded. The results of the impedance spectroscopy reveal an alternating behavior. The charge transfer processes are subdivided into a fast regime $(\tau<1 \mathrm{~s})$ and a slow regime $(\tau>1 \mathrm{~s})$. Both charge transfer resistances (CTR) are higher in the delithiated state than in the lithiated state. The amplitude is higher for the slow relaxation processes. The fast relaxation processes around $1 \mathrm{kHz}$ are attributed to the lithium electrode ${ }^{49-51}$. The slow relaxation processes take place mainly at the silicon electrode and correspond to the formation and dissolution of an inhibition layer ${ }^{34}$. Also, the solution resistance shows an alternating behavior. The solution resistance is decreased and increased in the lithiated and delithiated state, respectively. This can be explained by the growth of a layer on the electrodes, which introduces additional resistance, as well as, the charge-state dependent conductivity of lithiated silicon ${ }^{52}$. Also, the different mobility of lithium within lithiated and non-lithiated silicon influences the resistance ${ }^{53}$. An electrolyte decomposition process would be accompanied by a constant increase in the solution resistance. Nyquist plots of all states are shown in the supporting information (Fig. S2).

The size of the silicon crack pattern cells was determined in the final states of the $4^{\text {th }}$ to $8^{\text {th }}$ delithiation (Fig. 5). The total number of identified cells increases from approx. 1050 after the fourth cycle to 1878 within the $8^{\text {th }}$ delithiation. The analyzed area was $0.9 \times 1.3 \mathrm{~mm}$. However, the median area decreases from roughly $738 \mu \mathrm{m}^{2}$ to $572 \mu \mathrm{m}^{2}$ from the $4^{\text {th }}$ to the $5^{\text {th }}$ cycle, respectively. Afterwards, the median size stays nearly constant. Similar behavior is found if the first quartile is used for the data evaluation $\left(309 \mu \mathrm{m}^{2}\right.$ and $310 \mu \mathrm{m}^{2}$ after the $5^{\text {th }}$ and $8^{\text {th }}$ cycle, respectively). Therefore no significant continuous fracturization of the already formed cells occurs. The final state of the $8^{\text {th }}$ delithiation was analyzed further to get information about the length and width of the cells (Fig. S4)

4 in SI). The smallest box still encasing the individual cell was chosen as a size parameter. The shorter edge was taken as width, the longer one as the length of the box. The mean box length is $23.9 \mu \mathrm{m}$, whereas the mean box width is $16.3 \mu \mathrm{m}$, indicating a rectangular shape of the cells. Less than $5 \%$ of all cells are smaller than $6 \mu \mathrm{m}$ in width or $9 \mu \mathrm{m}$ in length. Due to the special morphology, the silicon electrode may behave more like silicon particles/rods than like a silicon layer and also lithiate anisotropically ${ }^{35,47,54}$. Therefore, even structures in the $\mu \mathrm{m}$-scale can withstand pulverization caused by mechanical fracturization. This behavior may originate from a limited lithiation degree ${ }^{20,40}$. Also, an anisotropic lithiation of the silicon patches may suppress the fracturization. However, also smaller, disordered cracks were reported elsewhere for different geometries ${ }^{21,30,55}$, which may be outside of the experimental resolution. Therefore, FIB/SEM was used for further analysis of the specimen. 


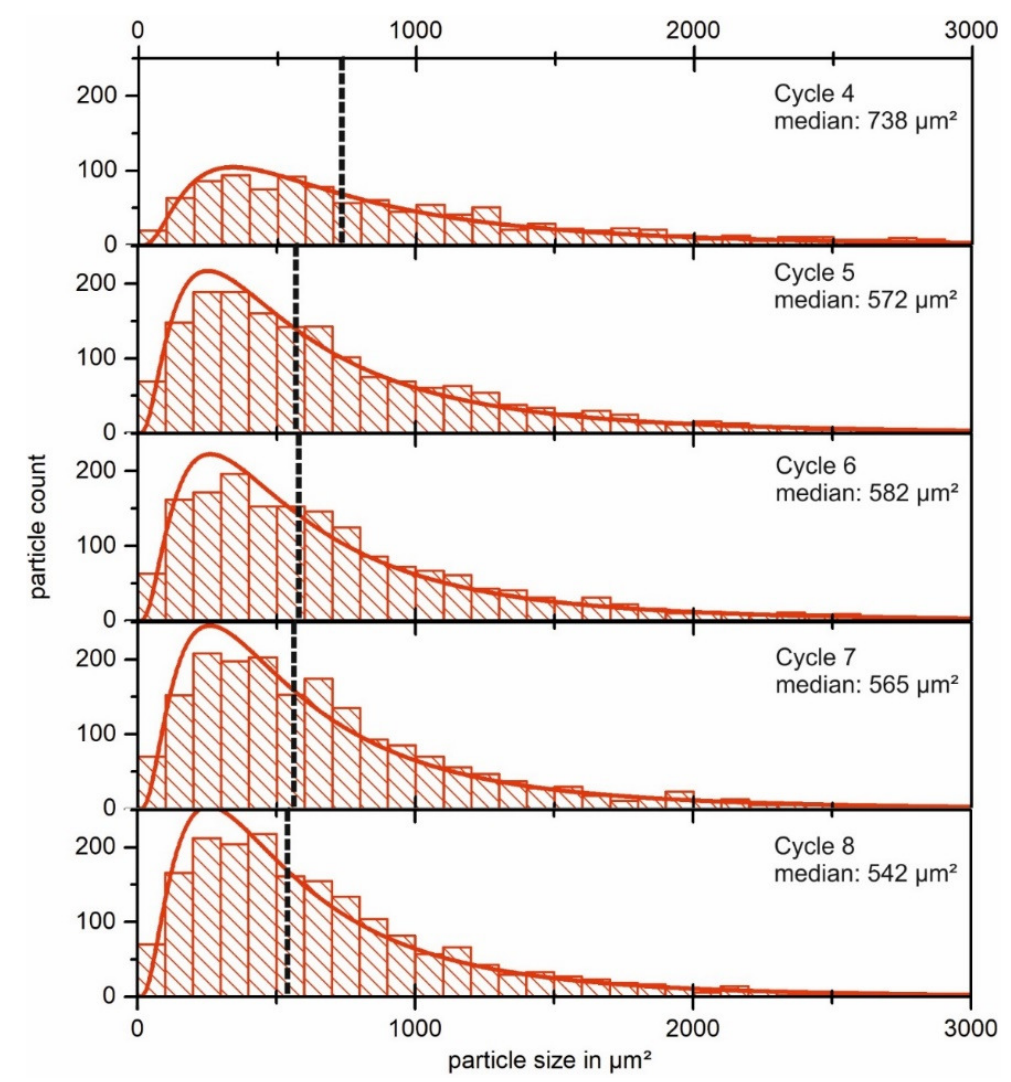

Fig. 5: Size distribution of the crack cells on the silicon electrode for the cycles 4-8. The size distribution can be described by a logarithmic normal distribution. The dashed line indicates the median area size. Areas larger than $3000 \mu \mathrm{m}^{2} \mathrm{were}^{2}$ treated as uncracked and therefore have been removed.

\section{$\underline{E x \text {-situ morphology analysis }}$}

Figure S5 shows an overview image of the final state of the cell after 15 cycles. Afterward, the cell was disassembled in the delithiated state, and the silicon electrode was placed in a focused ion beam/electron microscopy. The results are shown in figure 6. Clearly, a crack pattern can be found which follows the directions of the crystal planes (Fig. 4). This was proven by ex-situ Laue diffraction (Figure S6 in SI). As already mentioned, the crystalline silicon gets amorphous during the initial stage of the lithiation process ${ }^{4}$. Therefore, the cracking process starts already beforehand, since otherwise, no preferred direction should exist. Acoustic emission studies support these findings ${ }^{56}$. In the successive delithiation, the fractures become visible on the macroscale. No growth of fractures was observed during the lithiation process. This is in contrast to observations done by Bordes et al., who found fractures on micron-sized silicon particles during the first lithiation ${ }^{42}$.

The depth of the cracks is approximately $18 \mu \mathrm{m}$ (Fig. 6G). Also, cracks within the pillars were found, but they are thinner than the regular crack pattern and are not aligned with the crystallographic axes. Our findings are comparable to the study by Shi et al., who also found similar characteristic patterns in their ex-situ study ${ }^{30}$. Based on the depth of $18 \mu \mathrm{m}$ and a charge of $26 \mathrm{C}$ in the $15^{\text {th }}$ cycle $\left(16 \mathrm{C} / \mathrm{cm}^{2}\right)$, an average lithiation degree of 1.1 lithium atoms per silicon can be calculated. This is just a rough estimation, since the silicon most probably lithiates anisotropically and also the cracked depth not necessarily is similar to the thickness of the lithiated region. The estimation is by a factor of 2 smaller than values found in previous studies ${ }^{32-34,41,43}$. This could be explained by an inhomogeneous lithiation of the pattern cells since the electrochemical results indicate the formation of the highly lithiated silicon interphase $\left(\mathrm{Li}_{15} \mathrm{Si}_{4} \text { and } \mathrm{Li}_{22} \mathrm{Si}_{5}\right)^{4}$. 

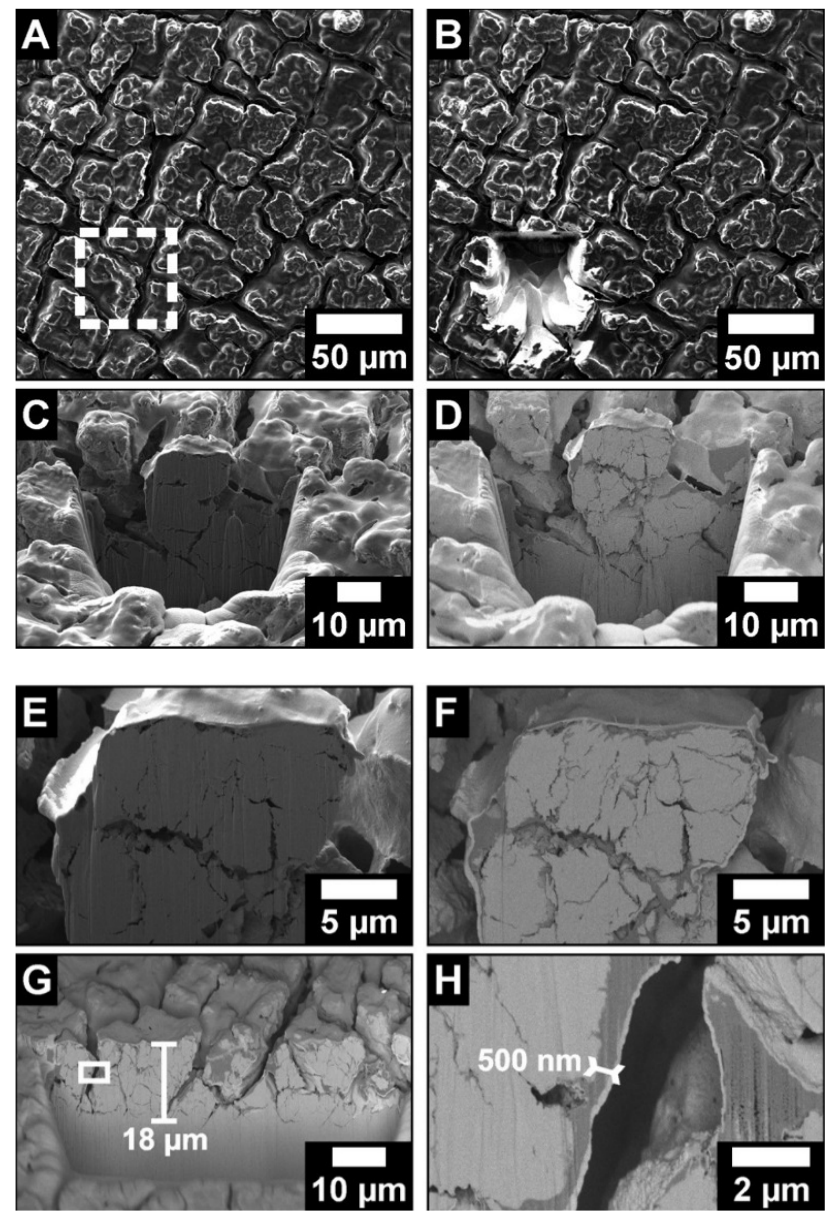

Fig. 6. Ex-situ FIB/SEM after 15 cycles. A) Overview image of the checkerboard pattern. B) FIB-cut of the marked area in A. C) \&D) magnified view of cut-area from b) using two different detectors (SE2-Detector and InLens BSE detector). E) \&F) magnified view of one pillar with two different detectors. G) Depth of cracks in the silicon shown in a broader cut-out region. H) Magnified view of the marked area in G) showing the thickness of a coating layer.

\section{CONCLUSION}

The morphological changes of a single crystal (100) silicon electrode during lithiation and delithiation were analyzed over eight cycles using operando phase-contrast radiography. The main results can be summarized as follows:

1. The time-resolved formation of an orthogonal fracturization pattern was observed. The crack pattern is aligned with the crystallographic axis of the crystalline silicon, as proved with Laue diffraction.

2. This pattern forms just after the first delithiation peak that corresponds to the decomposition of the highly lithiated phase, demonstrating certain robustness of the silicon anode towards induced mechanical stresses. The pattern vanishes during lithiation in the potentiostatic step. Therefore, capacity-limited cycling, which partly maintains the highly lithiated phase, may avoid the formation of fractures.

3. The cracks reappear in the same position again during the subsequent cycle, but also additional cracks can be found. Most likely, this is caused by the volume expansion of the patches during lithiation, which leads to physical contact between the patches, but the crack is still existent. No interspace between the patches exists in the lithiated space since this would introduce a signal in the phase-contrast radiography. This observation is only possible with operando experiments 
4. However, the area distribution of the fracture pattern cells stays nearly constant, meaning the individual cells can resist further fracturization.

Nevertheless, the continuous growth of the crack pattern may damage the electrode irreversibly at a certain point. Therefore long term operando experiments with even higher cycle numbers or a limited capacity operation of single-crystal silicon anodes could reveal further mechanical ageing mechanisms.

\section{EXPERIMENTAL}

We employed phase-contrast X-ray imaging to probe morphological changes in a lithium-silicon cell during cycling. The experiment is schematically shown in figure 1 and was performed at the BAMline end-station at BESSY II ${ }^{58}$. The measurement was performed in the phase-contrast regime using parallel, monochromatic, and coherent X-rays. The incoming beam gets deflected at differences in the refractive index (e.g., on material boundaries or cracks) as described by Snell's law of refraction. The refracted beam interferes with the regular X-rays, which highlights the physical boundaries mentioned beforehand. Therefore, the sample-detector distance needs to be adjusted according to the sample properties. An energy of $25 \mathrm{keV}$ was selected with the double multilayer monochromator. The detector system consists of a $60 \mu \mathrm{m}$-thick $\mathrm{CdWO}_{4}$ scintillator in combination with an optical peter lens system (20 times magnification) and a PCO.4000 camera (PCO AG) as shown in figure S7. The distance between the scintillator and the sample was $40 \mathrm{~cm}$ during this experiment. This imaging setup yields a $1.75 \mathrm{x} 1.17 \mathrm{~mm}^{2}$ field of view with a pixel size of $0.437 \mu \mathrm{m}$, and every frame was exposed for $60 \mathrm{~s}$. The sample environment was shifted out of the beam after every image, and a flat-field image was captured. This procedure results in a time resolution of 2.4 minutes per normalized radiogram. The total experiment time was 48.5 hours. Due to technical difficulties, the imaging process was paused after 20 hours for 1 hour. The electrochemistry was not stopped during that period. The missing 25 radiograms were added later via interpolation. In total, 1220 radiograms were recorded. The acquired images are a superposition of all cell parts, especially of the two electrodes and the separator.

The designed operando cell is shown in Fig. $7^{57}$. The X-ray-windows were made of aluminum, and the cell body was made of PEEK. All other parts were made of stainless steel. A 5-mm hole was drilled into both steel spacer to enhance X-ray transmission. Various ethylene propylene diene monomer rubber rings were used as sealing to avoid air and moisture contamination. Single crystal silicon disks with a polished (100) surface (n-doped, $500 \mu \mathrm{m}$ thickness, specific resistivity $10^{-3}$ to $40 \Omega \mathrm{cm}$, Sigma Aldrich) were used as the working electrode after cutting into $16 \mathrm{~mm}$ disks using a laser-cutting system. The silicon chip was immersed in 2-propanol (purity $>99.8 \%$, Sigma Aldrich, $\mathrm{H}_{2} \mathrm{O}<0.1 \%$ ) for $24 \mathrm{~h}$ to remove contaminations from the surface, such as dust or remains from the cutting process. A $1 \mathrm{M} \mathrm{LiPF}_{6}$ solution in ethylene
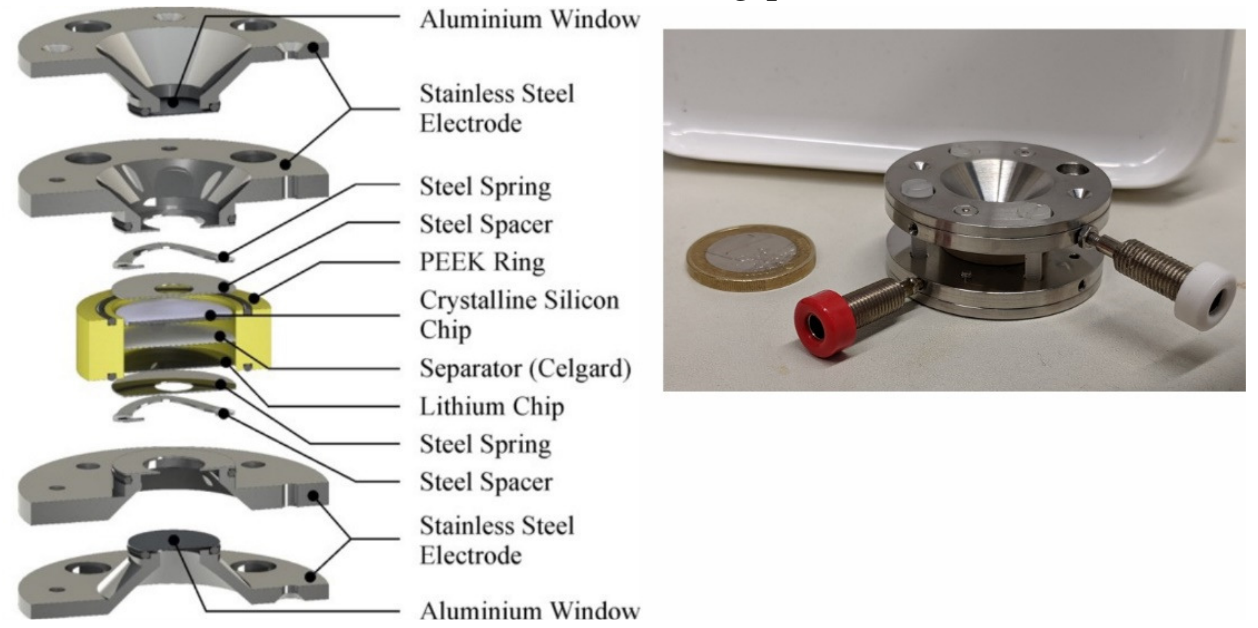

Fig. 7: Design of the operando cell used for the experiment ${ }^{57}$. On the left, a schematic view is shown, including the labeling of the different parts. On the right, a photo of the whole cell is shown together with a one euro coin for size 9 comparison. 
carbonate/dimethyl carbonate (1:1 by volume, battery grade, Sigma Aldrich) was used as the electrolyte. Metallic lithium foil serves as the counter electrode. A porous polymer foil (Celgard 2700) is employed as a separator. The cell was assembled in an argon-filled glovebox $\left(\mathrm{H}_{2} \mathrm{O}-\right.$ and $\mathrm{O}_{2}-$ level less than $\left.5 \mathrm{ppm}\right)$. A solution resistance of $4.5 \Omega$ was measured in the virgin state. The transmittance of the cell was roughly $50 \%$.

All of the image post-processing was done with Image J ${ }^{59}$. As the sample is moved for taking flat-fields, and the spatial resolution of the setup is below $1 \mu \mathrm{m}$, a drift was inevitable. This spatial shift was corrected using algorithms based on SIFT (Scale-Invariant Feature Transform) ${ }^{60}$. Due to the long exposure time and movement of the monochromator mirrors, a few horizontal stripes where still present in the normalized data. They were partially removed from the radiograms by a wavelet-based stripe filter ${ }^{61}$. The detailed procedure of image correction is described in figure S8. The stack of radiograms was divided by the median of the first ten radiograms to improve the contrast of the phase edges developing in the cell over time. Five radiograms (at times 23 h, 29 h, 35 h, 41 h, and 47 h, respectively) were selected where the cracks were most dominant. A new temporary stack was built with these images. The intensity of the phase shift at the cracks increased, and their position stayed the same. Since other structures were constantly changing in this temporary stack, the median image represents only the crack pattern. With a threshold it was then possible to build a mask representing the position of the cracks and thus calculating the crack refraction intensity over time. A random forest classifier was trained for the final retrieval of size distribution of the crack cells on the silicon ${ }^{62}$. Here the previously build mask for the crack positions was used for manual ground truth generation. The training set consisted of $0.3 \%$ of the whole dataset. A 10 -fold cross-validation was applied for training the 200 decision trees. The trained classifier was used to generate probability maps of the spatial crack distribution on the silicon electrode. At last a watershed was applied on the probability map resulting in the cycle dependent decomposition of the crack patterns. For the ex-situ FIB/SEM measurements, the silicon electrode was removed within an Ar-glovebox (H2O, $\mathrm{O} 2<1 \mathrm{ppm}$ ) from the cell and dried in vacuum at elevated temperatures. No rinsing with solvent was applied to avoid destroying the morphology of the silicon surface. The sample was sealed in a specialized, airtight sample shipper for the transfer into the FIB/SEM sample chamber. Here a short exposure to ambient conditions took place (approx.. 15 s). A ZEISS crossbeam 340 was used, which is equipped with a Gemini I electron gun and a Gallium ion source at an angle of $54^{\circ}$. First, the selected volume in the silicon electrode was sputtered with a gallium ion current of $15 \mathrm{nA}$ at $30 \mathrm{keV}$. For a final surface polishing, an ion current of $300 \mathrm{pA}$ at $30 \mathrm{keV}$ was applied. The milled surfaces have then been scanned using the SE2-Detector and InLens BSE detector with a low electron acceleration voltage of $1 \mathrm{keV}$. The scanning angle of $36^{\circ}$ has been corrected to achieve isotropic spatial dimensions.

For Laue-diffraction, an in-house built Laue camera with a Tungsten X-ray tube operating at $25 \mathrm{kV}$ was used. The data was recorded on a $12 \times 12 \mathrm{~cm}^{2}$ image plate, which is read out with a pixel size of $100 \mu \mathrm{m}$. The data were analyzed with the "OrientExpress" software. The brittle silicon wafer cracked before being removed from the cell. Therefore the angle between the crack and the top of the cell was determined. Afterwards, the silicon piece was glued on the sample table of the diffractometer with the breaking edge in the horizontal orientation

Electrochemical measurements were carried out using an interface 1000 potentiostat by GAMRY Instruments. First, impedance spectroscopy (EIS) at open circuit potential was performed (1 MHz to 0.1 $\mathrm{Hz}, 15 \mathrm{pts} /$ decade, $5 \mathrm{mV}$ root-mean-square (rms) amplitude) at open circuit potential (OCP) followed by a $1 \mathrm{~h}$-OCP-measurement for wetting the electrodes and alignment of the beamline instrument. Afterward, a linear voltage ramp was applied (starting at the last measured voltage, down to $15 \mathrm{mV}, 0.3 \mathrm{mV} \mathrm{s}-1$ ) followed by a potentiostatic step of $1 \mathrm{~h}$. At the same potential, an EIS was performed before ramping up the voltage to $2.0 \mathrm{~V}(0.3 \mathrm{mV} \mathrm{s}-1)$. Again, the potential was kept constant for $1 \mathrm{~h}$ followed by an EIS. The voltage-time-plot can be found in Fig. 4. Impedance data were analyzed using the equivalent circuit 
model, which is shown in the SI. Artifacts in the impedance data caused by the slower scan time at low frequencies were removed using the ZHiT-algorithm before analysis ${ }^{63}$.

\section{ACKNOWLEDGMENT}

We thank the beamline scientist, Dr. Bernd Müller, from BAMline for the technical support. We thank the Helmholtz-Zentrum Berlin for beamtime allocation. The help of Dr. Anna Manzoni and Dr. Konrad Siemensmeyer for the acquisition of the Laue-Diffraction images at the HZB Quantum Material Core Lab is gratefully acknowledged.

\section{REFERENCES}

(1) Larcher, D.; Tarascon, J.-M. M. Towards Greener and More Sustainable Batteries for Electrical Energy Storage. Nat. Chem. 2015, 7, 19-29.

(2) Feng, K.; Li, M.; Liu, W.; Kashkooli, A. G.; Xiao, X.; Cai, M.; Chen, Z. Silicon $\square$ Based Anodes for Lithium $\square$ Ion Batteries From Fundamentals to Practical Applications.Pdf. SMALL 2018, 14, 1702737-1702769.

(3) Ashuri, M.; He, Q.; Shaw, L. L. Silicon as a Potential Anode Material for Li-Ion Batteries: Where Size, Geometry and Structure Matter. Nanoscale 2016, 8, 74-103.

(4) McDowell, M. T.; Lee, S. W.; Nix, W. D.; Cui, Y. 25th Anniversary Article: Understanding the Lithiation of Silicon and Other Alloying Anodes for Lithium-Ion Batteries. Adv. Mater. 2013, 25, 4966-4985.

(5) Zuo, X.; Zhu, J.; Müller-Buschbaum, P.; Cheng, Y.-J. Silicon Based Lithium-Ion Battery Anodes: A Chronicle Perspective Review. Nano Energy 2017, 31, 113-143.

(6) Heubner, C.; Langklotz, U.; Michaelis, A. Theoretical Optimization of Electrode Design Parameters of Si Based Anodes for Lithium-Ion Batteries. J. Energy Storage 2018, 15, 181-190.

(7) Li, J.; Dahn, J. R. An In Situ X-Ray Diffraction Study of the Reaction of Li with Crystalline Si. J. Electrochem. Soc. 2007, 154, A156-A161.

(8) Wang, J. W.; He, Y.; Fan, F.; Liu, X. H.; Xia, S.; Liu, Y.; Harris, C. T.; Li, H.; Huang, J. Y.; Mao, S. X.; et al. Two-Phase Electrochemical Lithiation in Amorphous Silicon. Nano Lett. 2013, 13, 709-715.

(9) Liu, X. H.; Zhang, L. Q.; Zhong, L.; Liu, Y.; Zheng, H.; Wang, J. W.; Cho, J. H.; Dayeh, S. A.; Picraux, S. T.; Sullivan, J. P.; et al. Ultrafast Electrochemical Lithiation of Individual Si Nanowire Anodes. Nano Lett. 2011, 11, 2251-2258.

(10) McDowell, M. T.; Ryu, I.; Lee, S. W.; Wang, C.; Nix, W. D.; Cui, Y. Studying the Kinetics of Crystalline Silicon Nanoparticle Lithiation with in Situ Transmission Electron Microscopy. Adv. Mater. 2012, 24, 6034-6041.

(11) Liu, X. H.; Zhong, L.; Huang, S.; Mao, S. X.; Zhu, T.; Huang, J. Y. Size-Dependent Fracture of Silicon During Lithiation. ACS Nano 2012, 6, 1522-1531.

(12) Roiban, L.; Koneti, S.; Wada, T.; Kato, H.; Aires, F. J. C. S.; Curelea, S.; Epicier, T.; Maire, E. Three Dimensional Analysis of Nanoporous Silicon Particles for Li-Ion Batteries. Mater. Charact. 2017, 124, 165-170.

(13) Shen, C.; Ge, M.; Luo, L.; Fang, X.; Liu, Y.; Zhang, A.; Rong, J.; Wang, C.; Zhou, C. In Situ and Ex Situ TEM Study of Lithiation Behaviours of Porous Silicon Nanostructures. Sci. Rep. 2016, 6, 31334.

(14) Chevrier, V. L.; Dahn, J. R. First Principles Model of Amorphous Silicon Lithiation. J. 
Electrochem. Soc. 2009, 156, A454.

(15) Huang, S.; Zhu, T. Atomistic Mechanisms of Lithium Insertion in Amorphous Silicon. J. Power Sources 2011, 196, 3664-3668.

(16) Chan, M. K. Y.; Wolverton, C.; Greeley, J. P. First Principles Simulations of the Electrochemical Lithiation and Delithiation of Faceted Crystalline Silicon. J. Am. Chem. Soc. 2012, 134, 1436214374.

(17) Jerliu, B.; Hüger, E.; Dörrer, L.; Seidlhofer, B.-K.; Steitz, R.; Oberst, V.; Geckle, U.; Bruns, M.; Schmidt, H. Volume Expansion during Lithiation of Amorphous Silicon Thin Film Electrodes Studied by In-Operando Neutron Reflectometry. J. Phys. Chem. C 2014, 118, 9395-9399.

(18) Tardif, S.; Pavlenko, E.; Quazuguel, L.; Boniface, M.; Maréchal, M.; Micha, J. S.; Gonon, L.; Mareau, V.; Gebel, G.; Bayle-Guillemaud, P.; et al. Operando Raman Spectroscopy and Synchrotron X-Ray Diffraction of Lithiation/Delithiation in Silicon Nanoparticle Anodes. ACS Nano 2017, 11, 11306-11316.

(19) Zeng, Z.; Liu, N.; Zeng, Q.; Lee, S. W.; Mao, W. L.; Yi Cui. In Situ Measurement of Lithiation Induced Stress in Silicon Nanoparticles Using Micro-Raman Spectroscopy. Nano Energy 2016, 105-110.

(20) Ma, Z.; Li, T.; Huang, Y. L.; Liu, J.; Zhou, Y.; Xue, D. Critical Silicon-Anode Size for Averting Lithiation-Induced Mechanical Failure of Lithium-Ion Batteries. RSC Adv. 2013, 3, 7398.

(21) Dong, K.; Markötter, H.; Sun, F.; Hilger, A.; Kardjilov, N.; Banhart, J.; Manke, I. In Situ and Operando Tracking of Microstructure and Volume Evolution of Silicon Electrodes by Using Synchrotron $\mathrm{X} \square$ ray Imaging. ChemSusChem 2018, 12, 261-269.

(22) Zhao, C.; Wada, T.; De Andrade, V.; Gürsoy, D.; Kato, H.; Chen-Wiegart, Y. C. K. Imaging of 3D Morphological Evolution of Nanoporous Silicon Anode in Lithium Ion Battery by X-Ray NanoTomography. Nano Energy 2018, 52, 381-390.

(23) Paz-Garcia, J. M.; Taiwo, O. O.; Tudisco, E.; Finegan, D. P.; Shearing, P. R.; Brett, D. J. L.; Hall, S. A. 4D Analysis of the Microstructural Evolution of Si-Based Electrodes during Lithiation: TimeLapse X-Ray Imaging and Digital Volume Correlation. J. Power Sources 2016, 320, 196-203.

(24) Taiwo, O. O.; Heenan, T. M. M.; Finegan, D. P.; Brett, D. J. L.; Shearing, P. R.; Paz-García, J. M.; Hall, S. A.; Mokso, R.; Villanueva-Pérez, P.; Patera, A. Microstructural Degradation of Silicon Electrodes during Lithiation Observed via Operando X-Ray Tomographic Imaging. J. Power Sources 2017, 342, 904-912.

(25) Merla, Y.; Peled, E.; Tariq, F.; Lee, P. D.; Eastwood, D. S.; Biton, M.; Yufit, V.; Freedman, K.; $\mathrm{Wu}$, B.; Offer, G.; et al. In-Operando X-Ray Tomography Study of Lithiation Induced Delamination of Si Based Anodes for Lithium-Ion Batteries. ECS Electrochem. Lett. 2014, 3, A76A78.

(26) Sun, F.; Markötter, H.; Manke, I.; Hilger, A.; Alrwashdeh, S. S. Applied Surface Science Complementary X-Ray and Neutron Radiography Study of the Initial Lithiation Process in Lithium-Ion Batteries Containing Silicon Electrodes. Appl. Surf. Sci. 2017, 399, 359-366.

(27) Zielke, L.; Sun, F.; Markötter, H.; Hilger, A.; Moroni, R.; Zengerle, R.; Thiele, S.; Banhart, J.; Manke, I. Synchrotron X-Ray Tomographic Study of a Silicon Electrode Before and After Discharge and the Effect of Cavities on Particle Fracturing. ChemElectroChem 2016, 3, 11701177.

(28) Jana, M.; Singh, R. N. A Study of Evolution of Residual Stress in Single Crystal Silicon Electrode Using Raman Spectroscopy. Appl. Phys. Lett. 2017, 111, 0-5.

(29) Chon, M. J.; Sethuraman, V. A.; McCormick, A.; Srinivasan, V.; Guduru, P. R. Real-Time Measurement of Stress and Damage Evolution during Initial Lithiation of Crystalline Silicon. Phys. Rev. Lett. 2011, 107, 1-4. 
(30) Shi, F.; Song, Z.; Ross, P. N.; Somorjai, G. A.; Ritchie, R. O.; Komvopoulos, K. Failure Mechanisms of Single-Crystal Silicon Electrodes in Lithium-Ion Batteries. Nat. Commun. 2016, 7 , $1-8$.

(31) Heo, J. Y.; Chu, Y. Y.; Kang, C. S.; Choi, Y. S.; Oh, K. H.; Kim, Y.-U.; Lee, S.-H.; Cho, J. S.; Son, S.-B.; Suh, S.-S.; et al. Electrochemically Induced and Orientation Dependent Crack Propagation in Single Crystal Silicon. J. Power Sources 2014, 267, 739-743.

(32) Seidlhofer, B.-K.; Jerliu, B.; Trapp, M.; Hüger, E.; Risse, S.; Cubitt, R.; Schmidt, H.; Steitz, R.; Ballauff, M. Lithiation of Crystalline Silicon As Analyzed by Operando Neutron Reflectivity. ACS Nano 2016, 10, 7458-7466.

(33) Cao, C.; Steinrück, H. G.; Shyam, B.; Toney, M. F. The Atomic Scale Electrochemical Lithiation and Delithiation Process of Silicon. Adv. Mater. Interfaces 2017, 4, 1-7.

(34) Ronneburg, A.; Trapp, M.; Cubitt, R.; Silvi, L.; Cap, S.; Ballauff, M.; Risse, S. Surface Structure Inhibited Lithiation of Crystalline Silicon Probed with Operando Neutron Reflectivity. Energy Storage Mater. 2018, 18, 182-189.

(35) Pharr, M.; Zhao, K.; Wang, X.; Suo, Z.; Vlassak, J. J. Kinetics of Initial Lithiation of Crystalline Silicon Electrodes of Lithium-Ion Batteries. Nano Lett. 2012, 12, 5039-5047.

(36) Momose, A. Recent Advances in X-Ray Phase Imaging. 2005.

(37) Snigrev, A.; Snigreva, I.; Kohn, V.; Kuznetsov, S.; Schelokov, I. On the Possibilities of X $\square$ ray Phase Contrast Microimaging by Coherent High $\square$ energy Synchrotron Radiation. Rev. Sci. Instrum. 1995, 66, 5486-5492.

(38) Suzuki, Y.; Yagi, N.; Uesugi, K. X-Ray Refraction-Enhanced Imaging and a Method for Phase Retrieval for a Simple Object. J. Synchrotron Radiat. 2002, 9, 160-165.

(39) Jerliu, B.; Hüger, E.; Dörrer, L.; Seidlhofer, B. K.; Steitz, R.; Horisberge, M.; Schmidt, H. Lithium Insertion into Silicon Electrodes Studied by Cyclic Voltammetry and Operando Neutron Reflectometry. Phys. Chem. Chem. Phys. 2018.

(40) Obrovac, M. N.; Krause, L. J. Reversible Cycling of Crystalline Silicon Powder. J. Electrochem. Soc. 2007, 154, A103--A108.

(41) Jerliu, B.; Hüger, E.; Dörrer, L.; Seidlhofer, B. K.; Steitz, R.; Horisberger, M.; Schmidt, H. Lithium Insertion into Silicon Electrodes Studied by Cyclic Voltammetry and: Operando Neutron Reflectometry. Phys. Chem. Chem. Phys. 2018, 20, 23480-23491.

(42) Bordes, A.; De Vito, E.; Haon, C.; Boulineau, A.; Montani, A.; Marcus, P. Multiscale Investigation of Silicon Anode Li Insertion Mechanisms by Time-of-Flight Secondary Ion Mass Spectrometer Imaging Performed on an in Situ Focused Ion Beam Cross Section. Chem. Mater. 2016, 28, 15661573.

(43) Veith, G. M.; Doucet, M.; Baldwin, J. K.; Sacci, R. L.; Fears, T. M.; Wang, Y.; Browning, J. F. Direct Determination of Solid-Electrolyte Interphase Thickness and Composition as a Function of State of Charge on a Silicon Anode. J. Phys. Chem. C 2015, 119, 20339-20349.

(44) Michan, A. L.; Divitini, G.; Pell, A. J.; Leskes, M.; Ducati, C.; Grey, C. P. Solid Electrolyte Interphase Growth and Capacity Loss in Silicon Electrodes. J. Am. Chem. Soc. 2016, 138, 79187931.

(45) Shi, F.; Pei, A.; Boyle, D. T.; Xie, J.; Yu, X.; Zhang, X.; Cui, Y.; Thomas, D.; Xie, J.; Yu, X.; et al. Lithium Metal Stripping beneath the Solid Electrolyte Interphase. Proc. Natl. Acad. Sci. 2018, $115,8529-8534$.

(46) Liu, X. H.; Zheng, H.; Zhong, L.; Huang, S.; Karki, K.; Zhang, L. Q.; Liu, Y.; Kushima, A.; Liang, W. T.; Wang, J. W.; et al. Anisotropic Swelling and Fracture of Silicon Nanowires during Lithiation. Nano Lett. 2011, 11, 3312-3318. 
(47) Lee, S. W.; McDowell, M. T.; Choi, J. W.; Cui, Y. Anomalous Shape Changes of Silicon Nanopillars by Electrochemical Lithiation. Nano Lett. 2011, 11, 3034-3039.

(48) Long, B. R.; Chan, M. K. Y.; Greeley, J. P.; Gewirth, A. A. Dopant Modulated Li Insertion in Si for Battery Anodes: Theory and Experiment. J. Phys. Chem. C 2011, 115, 18916-18921.

(49) Aurbach, D. Impedance Spectroscopy of Lithium Electrodes Part 1 . General Behavior in Propylene Carbonate Solutions and the Correlation to Surface Chemistry and Cycling Efficiency. $J$. Electroanal. Chem. 1993, 348, 155-179.

(50) Aurbach, D.; Zaban, A. The Behaviour in Propylene Carbonate Solutions - The Significance of the Data Obtained. J. Electroanal. Chem. 1994, 367, 15-25.

(51) Risse, S.; Cañas;, N. A.; Wagner;Norbert; Härk;, E.; Ballauff, M.; Friedrich, K. A. Correlation of Capacity Fading Processes and Electrochemical Impedance Spectra in Lithium/Sulfur Cells. $J$. Power Sources 2016, 323, 107-114.

(52) Pollak, E.; Salitra, G.; Baranchugov, V.; Aurbach, D. In Situ Conductivity, Impedance Spectroscopy, and Ex Situ Raman Spectra of Amorphous Silicon during the Insertion/Extraction of Lithium. J. Phys. Chem. C 2007, 111, 11437-11444.

(53) Strauß, F.; Hüger, E.; Heitjans, P.; Geue, T.; Stahn, J.; Schmidt, H. Lithium Permeation through Thin Lithium-Silicon Films for Battery Applications Investigated by Neutron Reflectometry. Energy Technol. 2016, 1582-1587.

(54) Liu, X. H.; Zheng, H.; Zhong, L.; Huang, S.; Karki, K.; Zhang, L. Q.; Liu, Y.; Kushima, A.; Liang, W. T.; Wang, J. W.; et al. Anisotropic Swelling and Fracture of Silicon Nanowires during Lithiation. Nano Lett. 2011, 11, 3312-3318.

(55) Liu, X. H.; Zhong, L.; Huang, S.; Mao, S. X.; Zhu, T.; Huang, J. Y. Size-Dependent Fracture of Silicon Nanoparticles during Lithiation.-Supporting Information1. ACS Nano 2012, 6, 15221531.

(56) Rhodes, K.; Dudney, N.; Lara-Curzio, E.; Daniel, C. Understanding the Degradation of Silicon Electrodes for Lithium-Ion Batteries Using Acoustic Emission. J. Electrochem. Soc. 2010, 157, A1354.

(57) Risse, S.; Härk, E.; Kent, B.; Ballauff, M. Operando Analysis of a Lithium/Sulfur Battery by SmallAngle Neutron Scattering": 2019.

(58) Görner, W.; Hentschel, M. P.; Müller, B. R.; Riesemeier, H.; Krumrey, M.; Ulm, G.; Diete, W.; Klein, U.; Frahm, R. BAMline: The First Hard X-Ray Beamline at BESSY II. Nucl. Instruments Methods Phys. Res. Sect. A Accel. Spectrometers, Detect. Assoc. Equip. 2001, 467-468, 703-706.

(59) Schindelin, J.; Arena, E. T.; DeZonia, B. E.; Hiner, M. C.; Eliceiri, K. W.; Rueden, C. T.; Walter, A. E. ImageJ2: ImageJ for the next Generation of Scientific Image Data. BMC Bioinformatics 2017, $18,1-26$.

(60) Lowe, D. G. Distinctive Image Features from Scale-Invariant Keypoints. Int. J. Comput. Vis. 2004, 60, 91-110.

(61) Münch, B.; Trtik, P.; Marone, F.; Stampanoni, M. Stripe and Ring Artifact Removal with Combined Wavelet-Fourier Filtering. EMPA Act. 2009, 17, 34-35.

(62) Witten, I. H.; Frank, E.; Hall, M. a. Data Mining: Practical Machine Learning Tools and Techniques; 2011.

(63) Schiller, C. a.; Richter, F.; Gülzow, E.; Wagner, N. Validation and Evaluation of Electrochemical Impedance Spectra of Systems with States That Change with Time. Phys. Chem. Chem. Phys. 2001, 3, 374-378. 